condition that the integral $\int_{1}^{2}\left(\log \left(-1 / y_{1}^{\prime}\right)-1\right) d x$ take an extreme value is

$$
y_{1}^{\prime \prime}=0
$$

the substitution (3) is a member of the defined type and $F_{y^{\prime} y^{\prime}} \div F_{y y^{\prime}} \equiv y^{\prime}=$ const. is an integral of $(2) . y^{\prime}=c$, $\pi=\partial F^{\prime} \partial y^{\prime}=-y^{\prime}$ gives $\pi=-c$ for an integral of $(1)$.

CoRNell UNIVERSITY, August, 1907.

\title{
THE MAXIMUM VALUE OF A DETERMINANT.
}

BY DR. F. R. SHARPE.

HADAMARD * has shown that the maximum value of a determinant when the absolute value of each element does not exceed 1 is $n^{\frac{1}{2} n}$. The square of such a maximum determinant is a determinant having all its elements 0 except those of the principal diagonal. If the elements are restricted to real values, they are each \pm 1 and are so arranged that when compared row with row there is always an equal number of changes and permanences of sign amongst the corresponding elements. Hence $n$ is necessarily even. If we compare any two rows with a third row, the division of changes and permanences is again even. Hence $n$ must be a multiple of 4 . By a rearrangement of signs and order of columns we can always arrange any three rows in the form which for the case of $n=12$ is

$$
\begin{array}{rrrrrrrrrrrr}
1 & 1 & 1 & 1 & 1 & 1 & 1 & 1 & 1 & 1 & 1 & 1 \\
1 & 1 & 1 & 1 & 1 & 1 & -1 & -1 & -1 & -1 & -1 & -1 \\
1 & 1 & 1 & -1 & -1 & -1 & 1 & 1 & 1 & -1 & -1 & -1
\end{array}
$$

The actual maximum determinant is known for the following cases: (1) $n$ a power of 2 , (2) $n=12$ or 20 , (3) when the factors of $n$ are any of the preceding numbers. For example, when $n$ is 8 , the determinant is

* Bull. des Sciences math., 1893. 


$$
\left|\begin{array}{rrrrrrrr}
1 & 1 & 1 & 1 & 1 & 1 & 1 & 1 \\
1 & 1 & 1 & 1 & -1 & -1 & -1 & -1 \\
1 & 1 & -1 & -1 & 1 & 1 & -1 & -1 \\
1 & 1 & -1 & -1 & -1 & -1 & 1 & 1 \\
1 & -1 & 1 & -1 & 1 & -1 & 1 & -1 \\
1 & -1 & 1 & -1 & -1 & 1 & -1 & 1 \\
1 & -1 & -1 & 1 & 1 & -1 & -1 & 1 \\
1 & -1 & -1 & 1 & -1 & 1 & 1 & -1
\end{array}\right|=4096
$$

The object of this note is to determine the probable maximum value which the determinant actually attains when $n$ is not a multiple of 4 , and to prove certain properties of a determinant which does attain the maximum value $n^{\frac{1}{2} n}$ for real values of the elements.

Theorem I. The cofactor of any element of a maximum determinant of order $4 p$ and value $(4 p)^{2 p}$ is $\pm(4 p)^{2 p-1}$, the sign being the same as the sign of that element.

This follows at once from the form of the square of the determinant and the fact that if we combine the cofactors of one row with the elements of any other row the result is 0 .

TheOREM II. The second minors of a maximum determinant of order $4 p$ and value $(4 p)^{2 p}$ are $\pm 2(4 p)^{2 p-2}$ or 0 , according as the complementary minor is 2 or 0 .

Arrange the determinant as explained above and consider the minor of the first element of the first row. The cofactors of its first row are clearly 0 for the first $2 p-1$ elements and $2(4 p)^{2 p-2}$ for the last $2 p$ elements, because of the equal distribution of the signs of the elements of the last $2 p$ columns.

THEOREM III. The third minors of a maximum determinant of order $4 p$ and value $(4 p)^{2 p}$ are $\pm 4(4 p)^{2 p-3}$ or 0 , according as the complementary minor is 4 or 0 .

The determinant being arranged as in the previous theorem, consider the second minor obtained by omitting the first and second rows and the first and $(2 p+1)$ th columins. The cofactors of its first row are clearly 0 for the first $p-1$ elements, $-4(4 p)^{2 p-3}$ for the next $p$ elements and the last $p$ elements, and 0 for the remaining $p-1$ elements, because of the equal distribution of signs among the second $p$ and last $p$ columns of the original determinant. 
Theorem IV. If the sign of any element of a maximum determinant of order $4 p$ and value $(4 p)^{2 p}$ is changed and its row added to the original determinant together with a suitably chosen column, a determinant of order $4 p+1$ is formed with a value. $(16 p-4)(4 p)^{2 p-1}$.

Expand the new determinant in terms of the elements of the new column, the signs of which are to be chosen so that the the $4 p+1$ determinants obtained are all positive. We obtain the original determinant, the original determinant with a change of sign in one element, and $4 p-1$ other determinants with two rows identical except in the sign of one element. Hence the new determinant has the value

$(4 p)^{2 p}+(4 p)^{2 p}-2(4 p)^{2 p-1}+2(4 p-1)(4 p)^{2 p-1}=(16 p-4)(4 p)^{2 p-1}$.

Using Theorems I, II and IV, we see that at any rate for $p=1,2,3,4$, or 5 determinants exist

$$
\begin{aligned}
& \text { of order } 4 p-2, \text { value } 2(4 p)^{2 p-2} ; \\
& \text { of order } 4 p-1, \text { value }(4 p)^{2 p-1} ; \\
& \text { of order } 4 p, \text { value }(4 p)^{2 p} ; \\
& \text { of order } 4 p+1, \text { value }(16 p-4)(4 p)^{2 p-1} \text {. }
\end{aligned}
$$

These give for

$$
n=2,3, \quad 4, \quad 5, \quad 6, \quad 7, \quad 8, \cdots,
$$

values $2,4,16,48,128,512,4096, \ldots, 4294967296$.

Professor E. W. Davis's formula * $(n-2) 2^{n-1}$ gives for

$$
n=3, \quad 4,5, \quad 6, \quad 7, \quad 8, \cdots, \quad 16
$$

values $4,16,48,128,320,768, \ldots, 458752$.

This formula is clearly too small for $n>6$; in fact, when $n$ is a power of 2 above 5, Hadamard's value is greater than Davis's for the next higher power of 2 , and the former's value for $n=24$ is larger than the latter's for $n=2^{5}$.

\section{Cornelu University,} October, 1907.

\footnotetext{
* Bulletin, October, 1907.
} 